\title{
Percepção do ruído ocupacional e perda auditiva em estudantes de Odontologia
}

\author{
Markelane Santana Silva*; Daylana Pacheco da Silva**; Eduarda Sales Leal**; Anne Grazielle Lopes \\ Carvalho*; Paula Anaíde Leal de Miranda*; Carlos Alberto Monteiro Falcão \\ * Departamento de Odontologia Preventiva e \\ Social da Faculdade de Odontologia da \\ Universidade Estadual do Piauí, Parnaíba, Brasil \\ ** Departamento de Saúde Coletiva da Faculdade \\ de Odontologia da Universidade Estadual do \\ Piauí, Parnaíba, Brasil \\ *** Professor doutor no Departamento de \\ Odontologia Preventiva e Social da Faculdade \\ de Odontologia da Universidade Estadual do \\ Piauí, Parnaíba, Brasil
}

\section{RESUMO}

Este estudo teve como objetivo analisar a percepção do ruído ocupacional e a perda auditiva em estudantes de Odontologia de uma instituição pública. $\mathrm{O}$ estudo transversal foi realizado em Parnaíba, Brasil, em uma amostra intencional com alunos a partir do terceiro ano do curso $(n=62)$. Os dados coletados em questionários autoaplicáveis foram analisados pelos Testes do Quiquadrado de Pearson e Exato de Fisher. Os estudantes consideraram o ruído ocupacional nocivo à saúde $(82,3 \%)$ e o cirurgião-dentista susceptível à perda auditiva $(88,7 \%)$. As queixas mais relatadas foram cefaleia constante $(35,5 \%)$ e irritação/nervosismo (32,3\%). Medidas de prevenção não são utilizadas (98,4\%), apesar de serem conhecidas $(75,8 \%)$. Os estudantes já receberam informações sobre a perda auditiva $(53,2 \%)$, a maioria na própria Instituição de Ensino Superior (81,8\%). Audiometria nunca foi realizada $(80,6 \%)$. A legislação sobre tolerância ao ruído não é conhecida $(77,4 \%)$ e este conhecimento foi estatisticamente associado com o período em curso $(\mathrm{p}=0,004)$. Os estudantes de Odonto- logia desta instituição conhecem as consequências e medidas de prevenção, embora não as utilizem.

Descritores: Ruído Ocupacional. Perda Auditiva Induzida por Ruído. Estudantes de Odontologia.

\section{INTRODUÇÃO}

Os riscos ocupacionais presentes no cotidiano do cirurgião-dentista (CD) acarretam graves danos à saúde. A rotina de trabalho intensa sem condições ideais afeta a saúde física, produtividade e estado psicológico, principalmente em relação aos ruídos e ergonomia ${ }^{1}$.

O ruído é um agente físico de risco presente em diversos ambientes de trabalho. Suas repercussões e formas de controle têm sido objeto de crescentes estudos com forte impacto no âmbito da saúde pública ${ }^{1-3}$. A exposição ao ruído intenso é uma ameaça ocupacional e origina problemas que ultrapassam os prejuízos para a audição e afetam a qualidade de vida, tais como irritabilidade, estresse, cefaleias constantes, perturbação do sono, acidentes no trabalho, 
hipertensão, doença cardiovascular e ainda prejudicam tarefas que exijam concentração, velocidade e precisão de movimentos ${ }^{1,4-7}$.

A Norma Regulamentadora $\mathrm{n}^{\circ} 15$ $(1978)^{8}$ estabelece limites de tolerância para o ruído contínuo ou intermitente, tendo como base o limite de 8 horas para o nível de $85 \mathrm{~dB}$ decibéis $(\mathrm{dB})$. No entanto, acima de $40 \mathrm{~dB}$ podem surgir problemas como irritação e perda de concentração. Em universidades, a acústica ambiente das aulas práticas é caracterizada pelo ruído excessivo. $\mathrm{O}$ uso simultâneo de diversos aparelhos aumenta a intensidade total dos ruídos e exacerba o limite de tolerância ${ }^{9,10}$.

A Perda Auditiva Induzida por Ruído (PAIR) é uma doença profissional silenciosa presente no meio odontológico e o segundo problema de saúde mais comum no mundo industrializado $^{7,11}$. É caracterizada por uma diminuição gradual da acuidade auditiva decorrente da exposição continuada a elevados níveis de pressão sonora, que aumenta com o tempo, é progressiva e irreversível, porém é passível de prevenção ${ }^{12}$.

As alterações iniciais da PAIR são detectáveis na audiometria a partir de três anos, dependendo do grau de exposição ${ }^{13}$. Isso mostra que os profissionais podem ser susceptíveis desde a graduação. A abordagem de temas relacionados à execução segura do trabalho odontológico na formação acadêmica visa contribuir para a conscientização dos riscos ocupacionais. Diante dos fatos, este trabalho teve como objetivo analisar a percepção do ruído ocupacional e perda auditiva em estudantes de Odontologia de uma instituição pública, no que tange às consequências e medidas de prevenção.

\section{METODOLOGIA}

Trata-se de um estudo transversal realizado em uma Instituição de Ensino Superior (IES) pública de Parnaíba, Piauí, Brasil. A IES foi escolhida por conveniência devido à facilidade de acesso para a coleta dos dados. Para selecionar estudantes com mais contato com o ruído ocupacional nas atividades do curso, foi adotado o método de amostragem intencional. $\mathrm{O}$ critério de inclusão foi estar devidamente matriculado no curso de odontologia a partir do terceiro ano. Foram excluídos da pesquisa os estudantes que não estavam matriculados em nenhuma disciplina do curso, desistentes e que estavam afastados por motivo de doença ou licença-maternidade no período da coleta. A amostra totalizou 63 alunos do quinto, sétimo, nono e décimo períodos porque, na referida instituição, o ingresso de turmas é anual e os blocos são alternados.

Os dados foram coletados por uma única pesquisadora devidamente treinada, por meio de questionário estruturado e autoaplicável, previamente testado em quem não fazia parte da amostra, com questões fechadas e abertas, mediante assinatura do Termo de Consentimento Livre e Esclarecido pelos participantes. A pesquisa foi previamente aprovada pelo Comitê de Ética em Pesquisa (CEP) da instituição em que foi realizada a pesquisa, com o número do parecer 39609. Os princípios regidos pelas normas nacionais e internacionais que regulam as pesquisas com seres humanos foram seguidos por este trabalho, assim respeitando as determinações da resolução 466/12 do Conselho Nacional de Saúde (CNS), garantindo a confidencialidade, anonimato e não utilização das informações em prejuízo dos indivíduos.

Os resultados foram catalogados e submetidos à análise descritiva da distribuição das frequências absolutas e relativas por meio de tabelas com número e percentual. Para verificar a possível associação entre as variáveis foi aplicado o Teste do Qui-quadrado de Pearson e para os casos em que as frequências observadas foram menores que 5, foi utilizado o Teste Exato de Fisher. O nível de significância adotado foi 5\%. O software Excel ${ }^{\circledR} 2010$ (Microsoft, Redmond, WA, EUA) foi utili- 
zado para catalogar os dados e o PASW-18® (IBM Analytics, Armonk, NY, EUA) para análise estatística a partir da criação de um banco de dados.

\section{RESULTADOS}

A amostra intencional formada por 63 estudantes teve taxa de resposta de 98,4\% $(n=62)$. A proporção foi similar entre o sexos feminino $(48,4 \%)$ e masculino $(51,6 \%)$ e a idade média foi 21,9 anos.

A distribuição de acordo com o período em curso, sexo e faixa etária é apresentada na tabela 1 .

Os estudantes consideraram o ruído ocupacional nocivo à saúde $(82,3 \%)$ e o cirurgião-dentista um profissional susceptível à PAIR (88,7\%). Não foi verificada associação significativa $(\mathrm{p}>0,05) \quad \mathrm{com}$ as variáveis sexo e período em curso (tabela 2 ).

A tabela 3 apresenta o conhecimento dos estudantes quanto às medidas de prevenção na exposição ao ruído, conhecidas por $75,8 \%$ deles e as queixas mais recorrentes após o atendimento. Nenhuma medida de prevenção é usada por $98,4 \%$, apenas um estudante lubrifica os instrumentos rotatórios antes e após o atendimento.

Informações sobre a PAIR foram repassadas em algum momento a 53,2\% dos universitários, dos quais $81,8 \%$ relataram ter recebido na própria IES. Nenhum exame de audição foi realizado por $80,6 \%$. A pequena parcela que realizou o exame relatou que foi apenas por rotina $(66,7 \%)$ ou sensação de perda de audição (33,3\%). Não foi verificada associação significativa $(\mathrm{p}>0,05)$ com as variáveis sexo e período em curso (tabela 4).

A legislação sobre a tolerância ao ruído (NR - 15) não é conhecida por $77,4 \%$ dos acadêmicos. Foi verificada associação significativa $(\mathrm{p}=0,004)$ com o período em curso, em que o grupo de acadêmicos do quinto e sétimo período conhecem mais a legislação que aqueles do nono e décimo (tabela 5).

Tabela 1. Distribuição dos estudantes de Odontologia segundo as variáveis período em curso, sexo e faixa etária.

\begin{tabular}{llc}
\hline \multicolumn{1}{c}{ Variável } & n & \% \\
\hline $\begin{array}{l}\text { Período em } \\
\text { curso }\end{array}$ & & \\
Quinto & 14 & 22,5 \\
Sétimo & 14 & 22,5 \\
Nono & 17 & 27,5 \\
Décimo & 17 & 27,5 \\
Total & 62 & 100,0 \\
Sexo & & \\
Feminino & 30 & 48,4 \\
Masculino & 32 & 51,6 \\
Total & 62 & 100,0 \\
Faixa etária (em & & \\
anos) & & \\
Até 22 anos & 43 & 69,4 \\
Acima de 22 anos & 19 & 30,6 \\
Total & 62 & 100,0 \\
\hline
\end{tabular}


Tabela 2. Distribuição dos estudantes de Odontologia e valor de $p$, segundo as variáveis sexo e período em curso, sobre considerar o ruído nocivo à saúde e o cirurgião-dentista susceptível à PAIR.

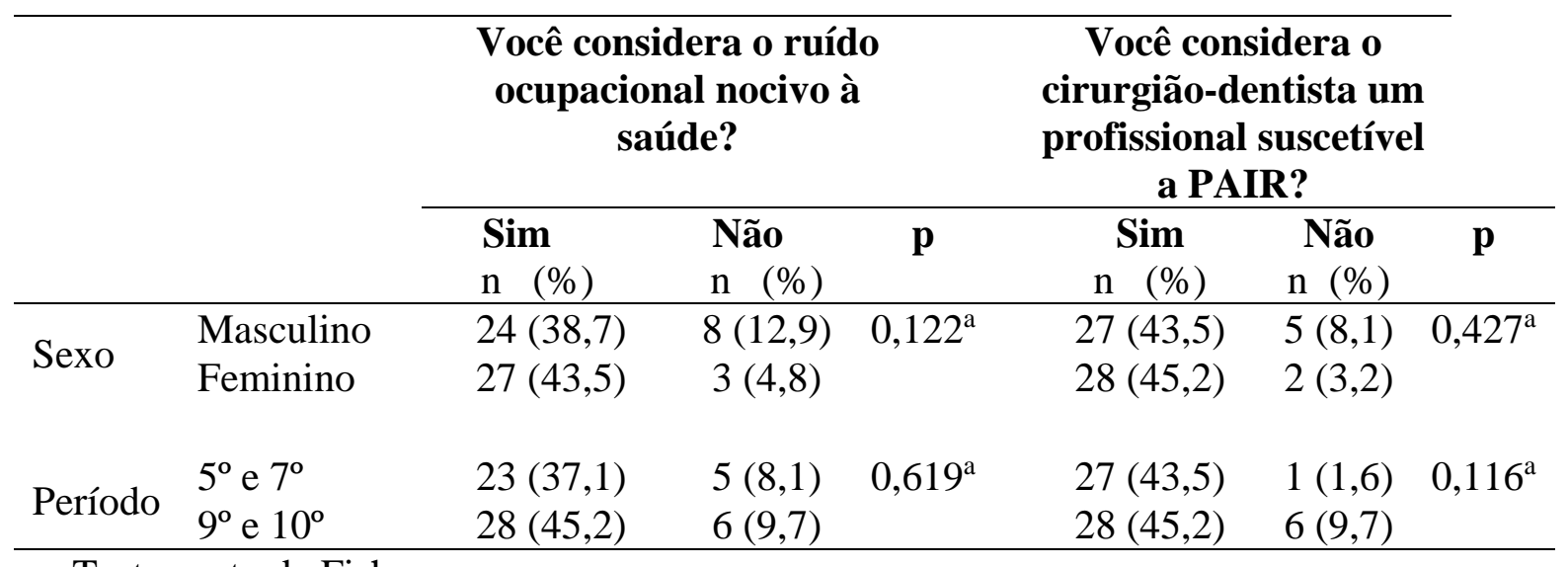

a: Teste exato de Fisher

Tabela 3. Frequência das medidas de prevenção e queixas após o atendimento citadas pelos estudantes de Odontologia.

\begin{tabular}{lcc}
\hline \multicolumn{1}{c}{ Variável } & n & \% \\
\hline Medidas de prevenção & 15 & 24,2 \\
\hline Não conhece nenhuma medida & 47 & 75,8 \\
Conhece alguma (s) medida (s) & 47 & 100,0 \\
\hline Protetor auricular & 21 & 44,7 \\
Instrumentos mais silenciosos & 8 & 17,0 \\
Isolamento acústico do compressor & 4 & 8,5 \\
Manutenção dos aparelhos & 2 & 4,2 \\
Som no consultório & & 35,5 \\
\hline Queixas após o atendimento & 22 & 32,3 \\
\hline Cefaleia constante & 20 & 25,8 \\
Irritação/nervosismo & 16 & 21,0 \\
Déficit de atenção & 13 & 11,3 \\
Dificuldade de comunicação & 7 & 9,7 \\
Dificuldades auditivas & 6 & \\
Zumbido & & \\
\hline
\end{tabular}


Tabela 4. Distribuição dos estudantes de Odontologia e valor de $p$, segundo as variáveis sexo e período em curso, sobre ter recebido informações relacionadas à PAIR e realizado exame de audição.

\begin{tabular}{|c|c|c|c|c|c|c|c|}
\hline & & \multicolumn{3}{|c|}{$\begin{array}{c}\text { Você recebeu informações } \\
\text { sobre a PAIR? }\end{array}$} & \multicolumn{3}{|c|}{$\begin{array}{l}\text { Você já realizou exame de } \\
\text { audição? }\end{array}$} \\
\hline & & $\begin{array}{l}\text { Sim } \\
\mathrm{n}(\%)\end{array}$ & $\begin{array}{l}\text { Não } \\
\mathrm{n}(\%)\end{array}$ & $\mathbf{p}$ & $\begin{array}{c}\text { Sim } \\
\mathrm{n}(\%)\end{array}$ & $\begin{array}{l}\text { Não } \\
\mathrm{n}(\%)\end{array}$ & $\mathbf{p}$ \\
\hline \multirow{2}{*}{ Sexo } & Masculino & $15(24,2)$ & $17(27,4)$ & 0,301 & $6(9,7)$ & $26(41,9)$ & 0,901 \\
\hline & Feminino & $18(29,0)$ & $12(19,4)$ & & $6(9,7)$ & $24(38,7)$ & \\
\hline \multirow{2}{*}{ Período } & $5^{\circ}$ e $7^{o}$ & $13(21,0)$ & $15(24,2)$ & 0,330 & $5(8,1)$ & $23(37,1)$ & 0,786 \\
\hline & $9^{\circ}$ e $10^{\circ}$ & $20(32,3)$ & $14(22,6)$ & & $7(11,3)$ & $27(43,5)$ & \\
\hline
\end{tabular}

Tabela 5. Distribuição dos estudantes de Odontologia e valor de $p$, segundo as variáveis sexo e período em curso, sobre conhecimento da legislação relacionada à tolerância ao ruído.

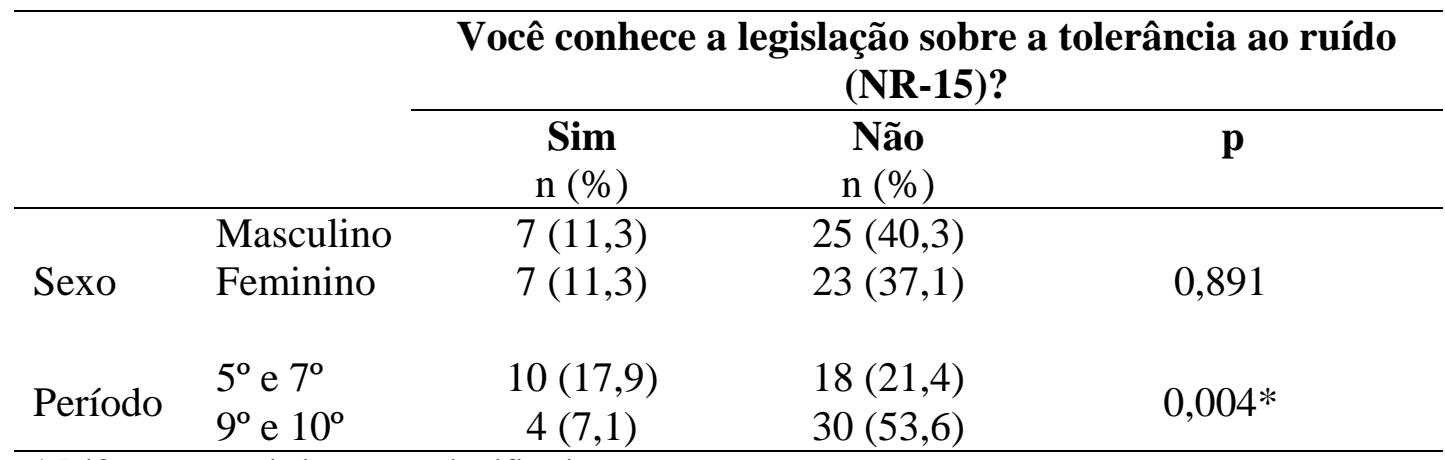

* Diferença estatisticamente significativa.

\section{DISCUSSÃO}

Por meio de dados provenientes de um estudo transversal foi analisada a percepção do ruído ocupacional em estudantes de odontologia no interior do Piauí, Brasil. Pesquisas anteriores também relataram investigações em estudantes de Odontologia $^{14,15}$, outras avaliaram o risco de o cirurgião-dentista apresentar danos auditivos $^{16-18}$. A prevalência da PAIR e alterações auditivas/extra auditivas em dentistas e universitários foi revelada em alguns estudos ${ }^{7,10,19}$. Al-Dujaili et al. $(2014)^{3}$ detectaram que os níveis de ruído em clínicas universitárias excederam os limites considerados nocivos à audição. Resultados contrários de Choosong et al. $(2011)^{20}$ e Brusis et al. (2008) $)^{21}$, que encontraram níveis de ruído abaixo do limite, não representando risco à PAIR.

As queixas relatadas pelos alunos foram semelhantes às de alguns estudos com profissionais, dentre elas cefaleia constante, déficit de atenção $^{22}$, irritação ${ }^{20}$, zumbido, intolerância a sons intensos, dificuldade de comunicação e estresse ${ }^{7}$. Isso prova que, mesmo com menor exposição, os sintomas 
podem estar presentes antes mesmo da formação.

Apesar de a maioria dos estudantes conhecer algumas medidas de prevenção, a lubrificação dos instrumentos rotatórios antes e após o atendimento é a única utilizada e apenas por um acadêmico. A exposição ocupacional a peças de mão de alta velocidade e outros dispositivos ruidosos pode ser um encargo adicional para a audição, mesmo que tenham reduzido consideravelmente o nível de ruído na última década ${ }^{21,23}$.

Um efeito de viés de confusão pode ter sido a causa do resultado dessa questão. Mais de $20 \%$ dos estudantes relataram não conhecer nenhuma medida de prevenção, e por isso podem ter respondido que não utilizam. A lubrificação dos instrumentos rotatórios pode ser realizada por uma boa parte dos estudantes sem saber que é um meio de reduzir a emissão de ruídos e prevenir a perda auditiva. Assim como o único estudante que realiza não a citou como um dos meios de prevenção.

Dentre os que conhecem as formas de prevenção, todos citaram o protetor auricular, mas nenhum o utiliza. Isso contradiz o fato de considerarem o ruído ocupacional nocivo à saúde e os dentistas susceptíveis à PAIR. Relato como o de Gambarra et al. $(2012)^{22}$ mostra que poucos dentistas usam o protetor auditivo. No estudo de Keenan (1999) ${ }^{18}$, os participantes da amostra justificaram a ausência do uso por falta de costume e no de Gonçalves et al. (2010) ${ }^{24}$ porque não era fornecido pela instituição de ensino. A desmotivação quanto ao uso do protetor auricular pode ser atribuída à falta do hábito entre os profissionais responsáveis pela formação acadêmica e ao fato de não estar inserido nos Equipamentos de Proteção Individual de uso obrigatório ${ }^{16,22}$.

Usar material fonoabsorvente, realizar manutenção de instrumentos rotatórios e aparelho de autoclave, realizar audiometria regularmente, disciplina no uso do sugador, evitar a permanência na sala de esterilização e estabelecer intervalos entre os atendimentos são outras medidas de prevenção e controle das doenças ocupacionais auditivas relatadas na literatura ${ }^{6,9,17,22}$

Assim como nesta investigação, Tôrres et al. $(2007)^{16}$ relataram que a maioria dos universitários receberam informações sobre PAIR na própria IES. No relato de Gonçalves et al. (2010) ${ }^{24}$ os profissionais relataram ter recebido orientações durante a graduação, mas não sabiam como se prevenir.

Embora essas informações tenham sido repassadas aos universitários, a maioria desconhece a legislação sobre a tolerância ao ruído. Foi verificada associação estatística significativa com o período em curso, sendo que o grupo do quinto e sétimo período conhecem mais a legislação. Isso pode ser justificado pelo fato de a disciplina que contempla os riscos ocupacionais no conteúdo, inclusive o ruído, ter sido cursada há menos tempo. Mesmo que o viés de memória possa ter influenciado esse resultado, nota-se a necessidade de enfatizar mais o assunto nas diversas disciplinas durante a graduação e incentivar a prática preventiva nas clínicas do curso.

Alguns estudantes relataram que realizaram o audiograma por sensação da perda de audição. Dificuldade auditiva também foi mencionada anteriormente como sintoma. Isso mostra que os estudantes já estão apresentando efeitos da exposição ao ruído ocupacional, possivelmente por não se prevenirem. Esses achados ressaltam a importância da prevenção a fim de evitar uma provável PAIR no futuro. A obtenção de um audiograma inicial para identificar a perda da audição ou verificar a audição normal com reavaliações periódicas é importante para monitorar a acuidade auditiva pessoal e a preservação do bem$\operatorname{estar}^{7,25}$.

Baseado nos resultados deste estudo, nota-se que o tema não é enfatizado na 
formação acadêmica. É essencial que o uso adequado das medidas de prevenção seja incentivado por responsáveis pela formação profissional. Programas de preservação auditiva voltados ao controle da emissão de ruídos dos equipamentos devem ser implantados desde a graduação. Além de preservar a saúde auditiva e a qualidade de vida, também melhora o desempenho e evita acidentes de trabalho ${ }^{6,16}$.

A pequena amostra intencional e de conveniência desta pesquisa pode não representar a realidade dos estudantes de Odontologia, contudo a alta taxa de resposta $(98,41 \%)$ contribuiu para a validade interna. $\mathrm{O}$ corte transversal com coleta em questionários autoaplicáveis possibilita a ocorrência de viés de memória e pode ser visto como uma limitação do estudo. No entanto, estudos transversais são essenciais para identificar indicadores de risco a serem investigados em outras avaliações como fatores de risco definitivos ${ }^{26}$. Estudos longitudinais com audiograma em amostras representativas para determinar os fatores de risco da PAIR e que identifiquem o nível de exposição ao ruído ocupacional são sugeridos para futuras pesquisas.

\section{CONCLUSÃO}

De acordo com a metodologia empregada, conclui-se que os estudantes de Odontologia da instituição pesquisada conhecem os riscos da exposição ao ruído ocupacional e sua relação com a perda auditiva. Os participantes têm ciência das medidas de prevenção, porém, não as utilizam. Não houve diferença estatística quanto ao sexo e período em curso em relação à percepção dos riscos auditivos e medidas de prevenção.

\section{AGRADECIMENTOS}

À Universidade Estadual do Piauí (UESPI) por ter financiado este estudo.

\section{ABSTRACT \\ Perception of occupational noise and hearing loss in dental students}

This study aimed to analyze the perception of occupational noise and hearing loss in dental students of a public institution. The crosssectional study was conducted in Parnaiba, Brazil, in intentional sample with of third year students $(n=62)$. Data collected through questionnaires were analyzed by Pearson's chi-square and Fisher's exact test. Students considered the occupational noise harmful to health $(82.3 \%)$ and the dentist susceptible to hearing loss $(88.7 \%)$. The most frequently reported complaints were constant headache $(35.5 \%)$ and irritation/nervousness (32.3\%). Preventative measures aren't used (98.4\%), despite being known (75.8\%). Students already have received information about hearing loss $(53.2 \%)$, most of on own institution of higher education (81.8\%). Audiometry has never been performed $(80.6 \%)$. The legislation concerning tolerance to noise isn't known $(77.4 \%)$ and this knowledge was statistically associated with the course period $(p=0,004)$. Dental students at this institution know the consequences and preventive measures, although don't use it.

Descriptors: Noise, Occupational. Hearing Loss, Noise-Induced. Students, Dental.

\section{REFERÊNCIAS}

1. Fernandes JC, Santos LN, Carvalho HJM. Avaliação de desempenho acústico de um consultório odontológico. Prod 2011; 21(3):509-17. [Acesso em: 15/06/2015] Disponível em: http://www.scielo.br/ scielo.php?script=sci_arttext\&pid=S0103 -65132011000300013

2. Stansfeld SA. Noise Effects on Health in the Context of Air Pollution Exposure. Int J Environ Res Public Health 2015; 12(10):12735-60. [Acesso em: 03/01 /2016] Disponível em: http://www.ncbi. nlm.nih.gov/pubmed/26473905 
3. Al-Dujaili M, Thomson WM, Meldrum R, Al-Ani AH. Noise levels in dental school clinics. N Z Dent J 2014; 110(3) :105-8. [Acesso em: 08/11/2015] Disponível em: http://www.ncbi.nlm.nih.gov/ pubmed/25265749

4. Basner M, Babisch W, Davis A, Brink M, Clark C, Janssen S, et al. Auditory and non-auditory effects of noise on health. Lancet 2014; 383(9925):1325-32. [Acesso em: 01/01/2015] Disponível em: http://www.ncbi.nlm.nih.gov/pmc/articles /PMC3988259/

5. Gurbuz MK1, Çatli T, Cingi C, Yaz A, Bal C. Occupational safety threats among dental personnel and related risk factors. J Craniofac Surg 2013; 24(6):e599-602. [Acesso em: 09/10/2014] Disponível em: http://www.ncbi.nlm.nih.gov/pubmed/242 20478

6. Dias A, Cordeiro R, Gonçalves CGO. Exposição ocupacional ao ruído e acidentes de trabalho. Cad Saúde Pública 2006; 22(10):2125-30. [Acesso em: 18/08/2014] Disponível em: http://www. scielo.br/scielo.php?pid=S0102-311X200 6001000018\&script=sci_arttext

7. Cavalcanti TLO, Andrade WTL. Efeitos auditivos e extra-auditivos decorrentes do ruído na saúde do dentista. $\mathrm{R}$ Bras $\mathrm{Ci}$ Saúde 2012; 16(2):161-6. [Acesso em: 22/02/2015] Disponível em: http://perio dicos.ufpb.br/ojs/index.php/rbcs/article/vi ew/11869

8. Brasil - Ministério do Trabalho e Emprego. Portaria 3.214 de jul. 1978. Normas regulamentadoras de segurança e saúde no trabalho (NR-15): Atividades e operações insalubres. [Acesso em: 07/ 02/2016] Disponível em: http://www.m te.gov.br/seguranca-e-saude-no-trabalho/ normatizacao/normas-regulamentadoras/ norma-regulamentadora-n-15-atividadese-operacoes-insalubres.

9. Saquy PC, Sousa Neto, MD, Felício, CM, Pécora, JD. Intensidade de ruído produzido pelas canetas de alta rotação.
RGO 1994; 42(3):131-3. [Acesso em: 05/03/2014] Disponível em: http:// bases.bireme.br/cgi-bin/wxislind.exe/iah/ online/?IsisScript=iah/iah.xis\&src $=$ googl e\&base $=$ BBO\&lang $=$ p\&nextAction $=$ lnk \&exprSearch=10093\&indexSearch=ID

10. Liu H, Zhu Xiaofang, Mo L, Peng X, Ni $\mathrm{X}$. [Cross-sectional study of hearing loss among freshmen in university]. Lin Chung Er Bi Yan Hou Tou Jing Wai Ke Za Zhi 2015; 29(18):1636-40. [Acesso em: 26/01/2016] Disponível em: http:// www.ncbi.nlm.nih.gov/pubmed/26790266

11. Khaimook W, Suksamae P, Choosong T, Chayarpham S, Tantisarasart R. The prevalence of noise-induced occupational hearing loss in dentistry personnel. Workplace Health Saf 2014; 62(9):35760. [Acesso em: 17/01/2015] Disponível em: $\quad$ http://www.ncbi.nlm.nih.gov/pub med/25650469

12. Comitê Nacional do Ruído e Conservação Auditiva. Boletim n. 6. [Acesso em 08/02/2016] Disponível em: http://www. arquivosdeorl.org.br/conteudo/acervo_por t.asp?id=125

13. Katz J. Tratado de audiologia clínica. 4. ed., São Paulo: Manole, 1999,465p.

14. Rovida TAS, Sumida DH, Santos AS, Moimaz SAS, Garbin CAS. Estresse e o estilo de vida dos acadêmicos ingressantes em um curso de graduação em odontologia. Rev ABENO. 2015; 15(3):26-34. [Acesso em: 08/02/2016] Disponível em: http://revabeno.emnu vens.com.br/revabeno/article/view/193/14

15. Marques MD, Souza CA, Pazos CTC, Amaral DS, Vieira EG, Campos WCS, et al. Expectativas dos estudantes de Odontologia quanto ao futuro profissional. Rev ABENO. 2015; 15(3):60-68. [Acesso em: 09/02/2016] Disponível em: http://revabeno.emnuve ns.com.br/revabeno/article/view/225/16

16. Tôrres BO, Fernandes MJM, Félix SSS, Costa ICC. A perda auditiva induzida pelo ruído (PAIR) na formação 
acadêmica: conhecimentos e medidas de prevenção. Odontol Clín-Cient 2007; 6(2):151-4. [Acesso em: 29/12/2014] Disponível em: http://bases.bireme.br/cgibin/wxislind.exe/iah/online/?IsisScript=ia $\mathrm{h} /$ iah. $x i s \& s r c=$ google $\&$ base $=$ BBO\&lang $=$ p\&nextAction $=$ lnk\&exprSearch $=28188 \&$ indexSearch=ID

17. Félix SSS. Análise do ruído ocupacional em odontologia: medidas de prevenção e implicações para a saúde de profissionais atuantes. [Tese]. João Pessoa: Universidade Federal da Paraíba; 2005.

18. Keenan VR. Ruído em consultório odontológico: dos riscos à prevenção. [Monografia]. Porto Alegre: Centro de Especialização em Fonoaudiologia Clínica; 1999. 79p. [Acesso em: 05/03/2014] Disponível em: http://www.cefac.br/library/teses/e2b3d55 a4fc3b0ab6140a2bdfa593a77.pdf

19. Gonçalves CGO, Marques JM, Ribas A, Lacerda ABM, Lobato DCB, Costa GL, et al. Caracterização dos limiares auditivos de odontólogos numa população da cidade de Curitiba - PR, Brasil. Arq Int Otorrinolaringol 2012; 16(1):32-38. [Acesso em: 12/03/2014] Disponível em: http://www.scielo.br/scielo.php?script=sci_ arttext\&pid=S1809-487220120001000 04

20. Choosong T, Kaimook W, Tantisarasart R, Sooksamear P, Chayaphum S, Kongkamol C, Srisintorn W, Phakthongsuk P. Noise Exposure Assessment in a Dental School. Saf Health Work 2011;2(4):348-54. [Acesso em: 15/05/2015] Disponível em: http:// www.ncbi.nlm.nih.gov/pubmed/22953219

21. Brusis T, Hilger R, Niggeloh $R$, Huedepohl J, Thiesen KW. [Are professional dental health care workers (dentists, dental technicians, assistants) in danger of noise induced hearing loss?] Laryngorhinootologie 2008; 87(5):33540. [Acesso em: 25/06/2015] Disponível em: http://www.ncbi.nlm.nih.gov/pub med/18058679
22. Gambarra PAN, Valença AMG, Cunha DGP. [The effects of occupational noise on hearing of dentists units of family's health of Joao Pessoa / PB]. Rev Bras Ciênc Saúde 2012; 16(3):361-70. [Acesso em: 19/09/2015] Disponível em: http:// bases.bireme.br/cgi-bin/wxislind.exe/iah /online/?IsisScript=iah/iah.xis\&src=googl e\&base $=$ LILACS\&lang $=$ p\&nextAction $=1$ nk\&exprSearch $=655246 \&$ indexSearch $=$ ID

23. Willershausen B, Callaway A, Wolf TG, Ehlers V, Scholz L, Wolf D, Letzel S. Hearing assessment in dental practitioners and other academic professionals from an urban setting. Head Face Med 2014; 10:1. [Acesso em: 02/11/2015] Disponível em: http://www.ncbi.nlm.nih.gov/pubmed/244 38539

24. Gonçalves CGO, Ribas A, Lacerda ABM, Gonçalves GA, Albizu E. A exposição ao ruído na prática da odontologia. In: Morata TC, Zucki FM(org). Saúde auditiva: avaliação de riscos e prevenção. São Paulo: Plexus, 2010.

25. Lopes AC, Melo ADP, Santos CC. Estudo dos limiares de audibilidade nas altas frequências em trabalhadores da área odontológica. Int Arq Otorrinolaringol 2012; 16(2):226-31. [Acesso em: 05/02/2016] Disponível em: http://www.scielo.br/scielo.php?script=sc i_arttext\&pid=S1809-48642012000200012

26. Lima-Costa MF, Barreto SM. [Types of epidemiologic studies: basic concepts and uses in the area of aging]. Epidemiol Serv Saúde 2003; 12(4):189-201. [Acesso em: 07/02/2016] Disponível em: http:// scielo.iec.pa.gov.br/scielo.php?script=sci _arttext\&pid=S1679-49742003000400003

Correspondência para:

Markelane Santana Silva

e-mail: markelanesantanasilva@gmail.com

Rua José Amâncio de Assunção, 120

64260-000 Santa Maria, Piripiri, PI 